\section{Visual dysfunction and its correlation with retinal changes in patients with Alzheimer's disease}

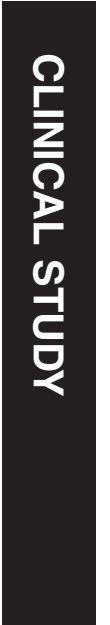

${ }^{1}$ Ophthalmology

Department, Miguel Servet University Hospital,

Zaragoza, Spain

${ }^{2}$ Aragon Health Research Institute (IIS Aragon-IACS), Zaragoza, Spain

Correspondence:

M Satue, Ophthalmology Department Miguel Servet University Hospital, Isabel la Catolica 1-3 C/ Padre Arrupe, Consultas Externas de Oftalmología, Zaragoza 50009 Spain

Tel: +0034976765558.

E-mail: mariasatue@ gmail.com

Received: 16 December 2015

Accepted in revised form: 24 January 2017

Published online: 10 March 2017

\begin{abstract}
Aim To evaluate visual dysfunction and its correlation with structural changes in the retina in patients with Alzheimer's disease (AD).

Methods Patients with AD $(n=24)$ and controls $(n=24)$ underwent evaluation of visual acuity (VA), color vision (using the Farnsworth and L'Anthony desaturated (D) 15 color tests), and contrast sensitivity vision (CSV; using the Pelli-Robson chart and CSV-1000E test) to measure visual dysfunction. Structural measurements of the retinal nerve fiber layer (RNFL) and macular thickness were obtained using spectral domain-optical coherence tomography (SD-OCT).

Results CSV at three of the four spatial frequencies was significantly worse in AD patients than in controls. Color vision was significantly affected in AD patients based on the Farnsworth color test. Compared with controls, macular thinning was detected in all sectors except the fovea, and the RNFL exhibited significant thinning in the superior quadrant and lower average thickness $(P<0.05)$. CSV was the functional parameter most strongly correlated with structural measurements in patients with $\mathrm{AD}$. Color vision was strongly associated with macular volume $(r>0.70$, $P<0.05)$. VA at different levels of contrast was associated with macular and RNFL thickness. Conclusions Patients with AD had visual dysfunction that correlated with structural changes evaluated by SD-OCT. Macular measurements may be reliable indicators of visual impairment in $\mathrm{AD}$ patients.
\end{abstract}

Eye (2017) 31, 1034-1041; doi:10.1038/eye.2017.23; published online 10 March 2017

\section{Introduction}

Alzheimer's disease (AD) is a progressive neurodegenerative disorder and the most
V Polo1,2, MJ Rodrigo ${ }^{1}$, E Garcia-Martin 1,2, S Otin 1,2, JM Larrosa ${ }^{1,2}$, MI Fuertes ${ }^{1,2}$, MP Bambo ${ }^{1,2}$, LE Pablo ${ }^{1,2}$ and M Satue ${ }^{1,2}$ frequent cause of dementia worldwide. ${ }^{1}$ Although AD is most commonly associated with memory deficits and cognitive impairment, patients with AD also exhibit alterations in sensory perception, such as visual processing. ${ }^{2-4}$

Recent studies reported retinal thinning in AD patients compared with healthy subjects, especially in the macular area. ${ }^{5-7}$ Defects in the retinal nerve fiber layer (RNFL) may be the earliest sign of $\mathrm{AD}$, even before damage to the hippocampus. ${ }^{8}$ These findings may reflect retinal ganglion cell death and axonal loss in the optic nerve beyond the effects of normal aging.

AD patients exhibit decreased color vision and contrast sensitivity, and other visual field deficits that could account for dysfunction in different tasks of basic vision and visual cognition in this disorder. ${ }^{9-11}$ Several studies report a correlation between visual dysfunction and axonal loss observed in the optic nerve in other neurodegenerative processes, such as multiple sclerosis and Parkinson disease. ${ }^{12,13}$ To our knowledge, however, very few published studies have assessed visual dysfunction in $\mathrm{AD}$ and its correlation with morphologic parameters. ${ }^{14}$

In the present study, we evaluated visual acuity (VA) using an Early Treatment Diabetic Retinopathy Study (ETDRS) chart, contrast sensitivity vision (CSV) using the CSV-1000E test and Pelli-Robson chart, and color vision using the Farnsworth and L'Anthony tests (by Color Vision Recorder software) in AD patients and healthy controls to examine the association between visual dysfunction and morphologic parameters.

\section{Materials and methods}

Twenty-four patients with mild or moderate AD and 24 age- and sex-matched healthy individuals were recruited for the study. 
All procedures adhered to the tenets of the Declaration of Helsinki, and the local ethics committee approved the experimental protocol. All participants provided informed consent to participate in the study. For cognitively impaired patients not able to give signed permission, legal tutors and family members provided signed consent, which was approved by the local ethics committee.

$\mathrm{AD}$ diagnosis was based on the Diagnostic and Statistical Manual of Mental Disorders, 4th edition (DSM-IV) established criteria for dementia syndrome (Alzheimer's type $)^{15}$ and the National Institute of Neurological and Communicative Disorders and Stroke and the Alzheimer's Disease and Related Disorders Association (NINDS-ADRDA) ${ }^{16}$ criteria for probable or possible AD. A trained neurologist blind to the visual and retinal test results evaluated all patients. The stage and severity of AD were determined by a trained neurologist using the MMSE scale, which includes items such as orientation, recall, calculation, registration, attention, repetition, comprehension, naming, reading, writing, and drawing. A score in of 30 on the MMSE scale indicates $100 \%$ correct. $^{17}$ Disease severity was determined by MMSE score as mild (18-24), moderate (10-18), or severe $(<9)$. Disease duration was also recorded. Patients with severe dementia and/or unable to perform the full exploration protocol due to their functional status were excluded from the study.

Inclusion criteria were confirmed AD diagnosis; best-corrected visual acuity (BCVA) of 0.4 or higher (using a Snellen chart) in each eye to allow for performance of the protocol; and intraocular pressure less than $21 \mathrm{~mm} \mathrm{Hg}$. Exclusion criteria were the presence of significant refractive errors ( $>5$ diopters of spherical equivalent refraction or 3 diopters of astigmatism); intraocular pressure $\geq 21 \mathrm{~mm} \mathrm{Hg}$; media opacifications; concomitant ocular diseases, including history of glaucoma or retinal pathology; and systemic conditions that could affect the visual system. Healthy controls had no history nor evidence of ocular or neurologic disease of any nature; their BCVA was $>20 / 30$ based on the Snellen scale. Only one eye per subject was randomly selected and included.

All subjects underwent a complete neuro-ophthalmic evaluation that included pupillary, anterior segment, and funduscopic examination. Visual function was assessed by evaluating BCVA using an ETDRS chart, CSV using the CVS-1000E test and Pelli-Robson chart, and color vision using the Farnsworth D15 and L'Anthony D15 tests. Structural analysis of the retina was performed using spectral domain (SD) optical coherence tomography (OCT) with the Cirrus high definition (HD) OCT (Carl Zeiss Meditec, Inc., Dublin, CA, USA), using two different applications: macular cube $512 \times 128$ applications (for macula thickness analysis) and optic disc cube $200 \times 200$ (for RNFL thickness analysis).

\section{Visual function evaluation}

LogMAR visual acuity (VA) was evaluated at three different contrast levels: 100\% (HCVA, using ETDRS chart), $2.50 \%$, and $1.25 \%$ (LCVA, using Low-Contrast Sloan Letter Charts, Precision Vision, LaSalle, IL, USA), the percentage indicating the level of contrast, that is, $100 \%$ representing black letters over white background and $1.25 \%$ light gray letters over white background. All measurements were obtained under monocular vision and controlled lighting conditions (photopic: mean luminance of $85 \mathrm{~cd} / \mathrm{m}^{2}$, high mesopic: $5 \mathrm{~cd} / \mathrm{m}^{2}$, and low mesopic: $3 \mathrm{~cd} / \mathrm{m}^{2}$ ) with best correction.

CSV was evaluated in our patients using the PelliRobson chart and the CVS-1000E test. The Pelli-Robson chart comprises horizontal lines of capital letters. The letter sequences are organized into groups of three (triplets) with two triplets per line. Within each triplet, all letters have the same contrast. The contrast decreases from one triplet to the next, even within each line. All patients were evaluated under monocular vision at a distance of 1 meter from the chart and under controlled photopic conditions $\left(85 \mathrm{~cd} / \mathrm{m}^{2}\right)$. The score corresponding to the last triplet of letters seen by the patient was recorded.

The CSV-1000E instrument is used worldwide for standardized CSV and glare testing. All patients were evaluated at a distance of $2.5 \mathrm{~m}$ from the chart under monocular vision at 4 different spatial frequencies $(3,6$, 12 , and 18 cycles per degree (cpd)). The chart comprised four rows of patches. Each row presents 17 circular patches 1.5 inches in diameter. The first patch on the far left in each row presents a very high contrast grating (sample patch). The remaining 16 patches appear in 8 columns across the row. In each column, one patch presents a grating and the other patch is blank. The patches that present a grating decrease in contrast from left to right across the row. The patient indicates whether the grating appears in the top patch or the bottom patch for each column. A CSV curve, in which the visual threshold is represented for each spatial frequency, was analyzed. Each contrast value for each spatial frequency was then transformed into a logarithmic scale according to standardized values.

Color vision was assessed using the Color Vision Recorder program (CVR, Optical Diagnostics, Beusichem, The Netherlands). The CVR software is designed for the Windows operating system and analyzes chromatic discrimination by color classification. The CVR includes the classic test of Farnsworth 100-hue, Farnsworth D15, and L'Anthony D15. All patients in the study were 
evaluated using the Farnsworth D15 and L'Anthony D15 protocols (often used to differentiate between subjects with severe loss of color vision and those with milder color defects or normal color vision) and different output parameters, such as the Age-Corrected Color Confusion index (AC CCI, which represents the ratio between the radius or distance between caps), the Confusion angle (Conf angle, which represents the axis of color deficiency), and the Scatter index (S-index, which represents the parallelism of confusion vectors to the personal confusion angle) were recorded. ${ }^{18,19}$ These parameters evaluate the severity of dyschromatopsia. A CCI score higher than 1 indicates altered color vision perception; the higher the score, the worse the condition. The tests were performed under monocular vision.

\section{Morphologic evaluation}

Structural measurements of the retina were obtained using the Cirrus OCT device. The same experienced operator performed all scans and did not apply manual correction to the OCT output. We used an internal fixation target because it provides the highest reproducibility ${ }^{20}$ and rejected poor-quality scans prior to data analysis. We based image quality assessment on the signal strength measurement, which combines the signalto-noise ratio with the uniformity of the signal within a scan (scale $1-10$, where 1 is categorized as poor image quality and 10 as excellent). We included images with a score higher than 7 for evaluation. The Cirrus OCT macular cube $512 \times 128$ application provides a macular volume measure and retinal thickness values for nine areas that correspond to the ETDRS. These areas include a central 1-mm circle representing the fovea, an inner ring measuring $3 \mathrm{~mm}$ in diameter, and an outer ring measuring $6 \mathrm{~mm}$ in diameter. The inner and outer rings are divided into four quadrants: superior, nasal, inferior, and temporal. The Cirrus OCT optic disc protocol generates $200 \times 200$ cubic images with 200 linear scans enabling analysis of the RNFL of a $6-\mathrm{mm}^{3}$ area around the optic nerve. For each scan series of RNFL measurements, we assessed the average, superior, inferior, temporal, and nasal thickness.

All data analyses were performed using SPSS software version 20.0 (SPSS, Inc., Chicago, IL, USA). The Kolmogorov-Smirnov test was used to assess sample distribution. Due to the normal distribution of the data, differences between evaluations of AD patients and healthy subjects were compared using the Student's $t$-test. The linear correlation between structural and functional parameters was determined using Pearson's correlation coefficient. Values of $\mathrm{p}$ less than 0.05 were considered to indicate statistical significance. To avoid a high false positive rate, the Bonferroni's correction for multiple tests was calculated and the corrected $P$-values were added to the previously calculated data.

\section{Results}

Twenty-four patients with AD (48 eyes) and 24 healthy controls (48 eyes) were included in the study. Mean age of the patients with $\mathrm{AD}$ was $74.42 \pm 8.88$ years and the mean age of the healthy controls was $72.94 \pm 7.40$ years. The two groups did not differ significantly in age $(P=0.070)$, sex $(P=0.670)$, or intraocular pressure $(P=0.540)$. Disease duration ranged from 12 months to 8 years with a median of 4.17 years since diagnosis. Mean MMSE score was $15.54 \pm 7.1$.

\section{Functional parameters}

CSV was affected in patients at three of the four spatial frequencies of the CSV-1000E chart $(3,6$, and $12 \mathrm{cpd})$ when analyzed based on the number of correct localized gratings $(P=0.020,<0.001$, and 0.010 respectively). The Pelli-Robson results also revealed a significant reduction in CSV in AD patients $(P=0.020)$. Color vision results were also significantly

Table 1 Visual function parameters in healthy controls and subjects with Alzheimer's disease

\begin{tabular}{lccc}
\hline Functional parameters & Healthy subjects & Alzheimer patients & $\mathrm{P}$ \\
\hline Visual acuity & & & \\
AV ETDRS 100 & $-0.04(0.08)$ & $0.10(0.08)$ & 0.940 \\
AV ETDRS 2.5 & $0.46(0.15)$ & $0.56(0.19)$ & 0.070 \\
AV ETDRS 1.25 & $0.56(0.17)$ & $0.714(0.21)$ & 0.150 \\
& & & \\
Contrast sensitivity & & & \\
Pelli-Robson & $1.90(0.10)$ & $1.57(0.18)$ & $\mathbf{0 . 0 2 0}$ \\
CSV 1000 3cpd & $1.71(0.13)$ & $1.45(0.22)$ & $\mathbf{0 . 0 2 0}$ \\
CSV 1000 6cpd & $1.94(0.15)$ & $1.67(0.33)$ & $\mathbf{0 . 0 0 0}$ \\
CSV 1000 12cpd & $1.61(0.17)$ & $1.23(0.33)$ & $\mathbf{0 . 0 1 0 ^ { * }}$ \\
CSV 1000 18cpd & $1.08(0.22)$ & $0.89(0.29)$ & 0.060 \\
& & & \\
Chromatic vision & $1.04(0.32)$ & $1.36(0.47)$ & $\mathbf{0 . 0 2 0}$ \\
CVR farnsw AC CCI & $63.92(11.33)$ & $65.59(8.70)$ & 0.950 \\
CVR farnswConfAngle & $1.56(0.21)$ & $2.04(0.46)$ & $\mathbf{0 . 0 0 0}$ \\
CVR farnsw S-index & $1.09(0.32)$ & $1.45(0.51)$ & $\mathbf{0 . 0 4 0 ^ { * }}$ \\
CVR lanthony AC CCI & $62.59(14.94)$ & $69.18(15.80)$ & 0.690 \\
CVR lanthonyConfAngle & $1.73(0.52)$ & $1.97(0.47)$ & 0.860 \\
CVR lanthony S-index & & & \\
\hline
\end{tabular}

Abbreviations: AC CCI, age-corrected color confusion index; Conf Angle, confusion angle; cpd, cycles per degree; ETDRS, early treatment diabetic retinopathy study; S-index, scatter index; VA, visual acuity.

Results are reported as mean (SD). Results in bold letters indicate statistical significance $(P<0.050)$. Asterisk indicates a significant difference by Student's $t$ test after Bonferroni correction for multiple tests $(P \leq 0.017$ for ETDRS; $P \leq 0.010$ for CSV 1000 E measurements; $P \leq 0.008$ for Farnsworth and L'Anthony tests). 
affected in AD (Farnsworth's AC CCI and S-Index, and L'Anthony's AC CCI). AD patients had a lower BCVA at all three contrast levels of the ETDRS chart compared to controls, but the results did not differ

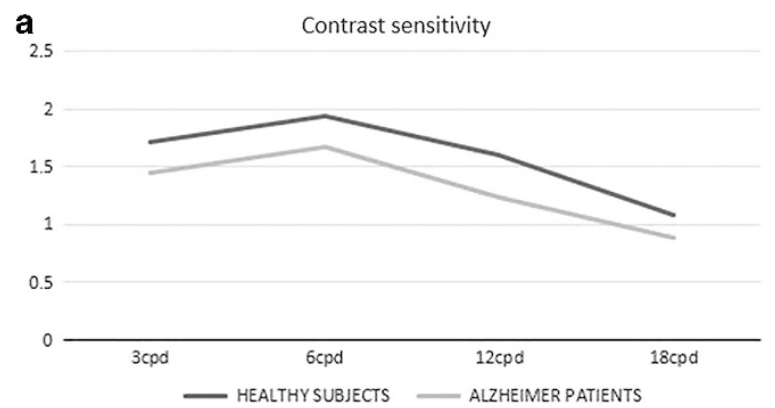

b

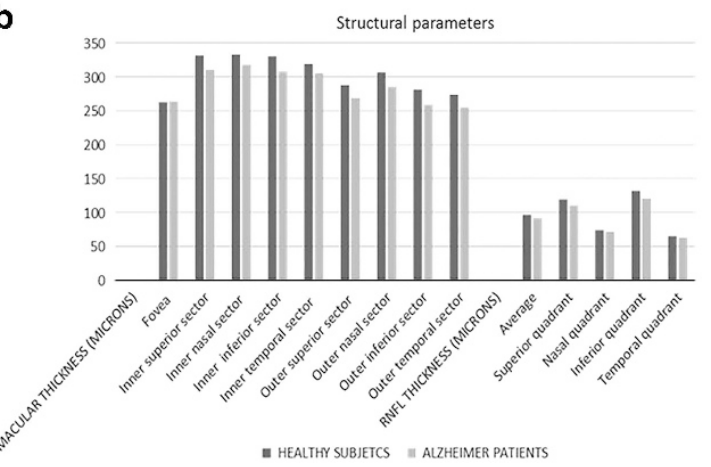

Figure 1 (a) Contrast sensitivity curve in patients with Alzheimer's disease and healthy controls. CPD, cycles per degree. (b) Structural parameters measured by OCT in patients with Alzheimer's disease and healthy controls. RNFL, retinal nerve fiber layer. significantly. The results are shown in Table 1 and Figure 1a.

\section{Structural parameters}

OCT measurements indicated a significant thickness reduction in $\mathrm{AD}$ patients in 8 of the 9 macular sectors analyzed (Table 2, Figure 1b). The RNFL was significantly reduced in the superior quadrant and the average thickness in AD patients (Table 2, Figure 1b).

\section{Correlation between functional and structural parameters}

CSV was the functional parameter most frequently associated with structural measurements in AD. The PelliRobson CSV results correlated with macular thickness in almost all sectors (Table 3$)$. Thickness in the outer superior $(r=0.65, \mathrm{p}<0.001)$, outer nasal $(r=0.58, \mathrm{p}<0.001)$, and outer temporal $(r=0.66, P<0.001)$ regions, and average thickness $(r=0.63, \mathrm{p}<0.001)$ were most highly correlated with CSV. The Pelli-Robson results also correlated with the RNFL thickness in different sectors (Table 3). Measurements with the CSV-1000E at different spatial frequencies correlated significantly with most macular parameters and with average and superior RNFL thicknesses (Table 3).

A significant but mild association between color vision (measured with both Farnsworth and L'Anthony tests) and the RNFL and macular parameters was found in isolated sectors $(r<0.50, P<0.05)$. Macular volume was

Table 2 Structural parameters obtained with the Cirrus HD optical coherence tomography device in healthy controls and subjects with Alzheimer's disease

\begin{tabular}{|c|c|c|c|}
\hline Structural parameters & Healthy subjetcs & Alzheimer patients & $\mathrm{P}$ \\
\hline \multicolumn{4}{|l|}{ Macular thickness } \\
\hline Fovea & $262.03(18.31)$ & $263.12(23.53)$ & 0.530 \\
\hline Inner superior sector & $331.65(13.06)$ & $310.44(27.58)$ & 0.010 \\
\hline Inner nasal sector & $333.42(13.33)$ & $317.19(21.44)$ & 0.010 \\
\hline Inner inferior sector & $330.97(12.34)$ & 307.75 (31.87) & $<0.001^{*}$ \\
\hline Inner temporal sector & 319.55 (13.19) & 305.94 (20.33) & 0.030 \\
\hline Outer superior sector & $288.23(9.34)$ & $269.63(21.57)$ & $<0.001^{*}$ \\
\hline Outer nasal sector & 306.39 (11.14) & $285.88(19.28)$ & $<0.001^{*}$ \\
\hline Outer inferior sector & $281.13(9.66)$ & $258.69(35.62)$ & 0.010 \\
\hline Outer temporal sector & $274.00(10.10)$ & $255.69(33.06)$ & $<0.001^{*}$ \\
\hline \multicolumn{4}{|l|}{ RNFL thickness } \\
\hline Average & $97.61(7.13)$ & $91.00(11.23)$ & $<0.001^{*}$ \\
\hline Superior quadrant & $119.29(10.53)$ & $110.06(20.71)$ & $<0.001^{*}$ \\
\hline Nasal quadrant & 74.03 (13.26) & $71.44(14.40)$ & 0.780 \\
\hline Inferior quadrant & $131.06(12.51)$ & $120.00(17.24)$ & 0.180 \\
\hline Temporal quadrant & $65.87(9.04)$ & $62.12(9.12)$ & 0.720 \\
\hline
\end{tabular}

Abbreviation: RNFL, retinal nerve fiber layer.

Results are reported as mean (SD). Results in bold letters indicate statistical significance $(P<0.050)$. Asterisk indicates a significant difference by Student's $t$ test after Bonferroni correction for multiple tests ( $P \leq 0.006$ for macular measurements; $P \leq 0.010$ for RNFL measurements). 
Table 3 Correlation between structural parameters (macular and retinal nerve fiber layer thickness) and contrast sensitivity vision (CSV) evaluated with Pelli-Robson and CSV-1000E tests in patients with Alzheimer's disease

\begin{tabular}{|c|c|c|c|c|c|c|c|c|c|c|}
\hline & \multicolumn{8}{|c|}{ CSV-1000E TEST } & \multicolumn{2}{|c|}{ Pelli Robson } \\
\hline & $6 C P D$ & P-value & $9 C P D$ & P-value & $12 C P D$ & P-value & $18 C P D$ & P-value & $\mathrm{R}$ & P-value \\
\hline Fovea & 0.043 & 0.770 & 0.19 & 0.900 & 0.45 & 0.760 & 0.14 & 0.330 & -0.02 & 0.881 \\
\hline Inner superior sector & 0.43 & 0.030 & 0.35 & 0.010 & 0.44 & 0.020 & 0.43 & 0.030 & 0.45 & $<0.001^{*}$ \\
\hline Inner nasal sector & 0.48 & 0.010 & 0.37 & 0.010 & 0.44 & 0.020 & 0.43 & 0.040 & 0.48 & $<0.001^{*}$ \\
\hline Inner inferior sector & 0.34 & 0.020 & 0.28 & 0.050 & 0.40 & $<0.001^{*}$ & 0.38 & 0.010 & 0.47 & $<0.001^{*}$ \\
\hline Inner temporal sector & 0.42 & 0.030 & 0.23 & 0.110 & 0.36 & 0.010 & 0.44 & $<0.001^{*}$ & 0.43 & $<0.001^{*}$ \\
\hline Outer superior sector & 0.50 & $<0.001^{*}$ & 0.36 & 0.010 & 0.57 & $<0.001^{*}$ & 0.50 & $<0.001^{*}$ & 0.65 & $<0.001^{*}$ \\
\hline Outer nasal sector & 0.50 & $<0.001^{*}$ & 0.50 & $<0.001^{*}$ & 0.49 & $<0.001^{*}$ & 0.50 & $<0.001^{*}$ & 0.58 & $<0.001^{*}$ \\
\hline Outer inferior sector & 0.17 & 0.240 & 0.27 & 0.050 & 0.38 & $<0.001^{*}$ & 0.32 & 0.030 & 0.38 & $<0.001^{*}$ \\
\hline Outer temporal sector & 0.47 & $<0.001^{*}$ & 0.22 & 0.120 & 0.50 & $<0.001^{*}$ & 0.39 & $<0.001^{*}$ & 0.66 & $<0.001^{*}$ \\
\hline Average thickness & 0.43 & 0.090 & 0.21 & 0.420 & 0.56 & 0.030 & 0.53 & 0.050 & 0.63 & $<0.001 *$ \\
\hline Macular volume & 0.48 & 0.050 & 0.34 & 0.150 & 0.30 & 0.240 & 0.27 & 0.320 & 0.54 & 0.010 \\
\hline Superior quadrant & 0.31 & 0.030 & 0.23 & 0.040 & 0.46 & $<0.001^{*}$ & 0.41 & $<0.001^{*}$ & 0.46 & $<0.001^{*}$ \\
\hline Nasal quadrant & 0.37 & $0.010^{*}$ & 0.19 & 0.200 & 0.20 & 0.190 & 0.11 & 0.440 & 0.37 & $0.010^{*}$ \\
\hline Inferior quadrant & 0.22 & 0.140 & 0.11 & 0.440 & 0.23 & 0.120 & 0.23 & 0.110 & 0.22 & 0.140 \\
\hline Temporal quadrant & 0.23 & 0.110 & 0.35 & $0.010^{*}$ & 0.26 & 0.740 & 0.35 & $0.010^{*}$ & 0.12 & 0.410 \\
\hline
\end{tabular}

Abbreviations: CSV, contrast sensitivity vision; RNFL, retinal nerve fiber layer.

Data in bold correspond to statistically significant correlations ( $P$-value $<0.05)$. Asterisk indicates a significant difference by Pearson's test after Bonferroni's correction for multiple tests ( $P \leq 0.0045$ for macular thickness; $P \leq 0.010$ for RNFL thickness).

Table 4 Correlation between structural parameters (macular and retinal nerve fiber layer thickness) and visual acuity in different contrast levels in patients with Alzheimer's disease

\begin{tabular}{|c|c|c|c|c|c|c|}
\hline Structural parameters & AV ETDRS $100 \%$ & P-value & AV ETDRS $2.5 \%$ & P-value & AV ETDRS $1.25 \%$ & P-value \\
\hline \multicolumn{7}{|l|}{ Macular thickness } \\
\hline Fovea & 0.21 & 0.150 & 0.22 & 0.140 & 0.17 & 0.230 \\
\hline Inner superior sector & 0.25 & 0.090 & 0.19 & 0.190 & 0.30 & 0.040 \\
\hline Inner nasal sector & 0.18 & 0.210 & 0.12 & 0.410 & 0.18 & 0.210 \\
\hline Inner inferior sector & 0.33 & 0.020 & 0.21 & 0.150 & 0.26 & 0.070 \\
\hline Inner temporal sector & 0.16 & 0.280 & 0.09 & 0.540 & 0.19 & 0.200 \\
\hline Outer superior sector & 0.46 & $<0.001^{*}$ & 0.36 & 0.010 & 0.45 & $<0.001^{*}$ \\
\hline Outer nasal sector & 0.40 & $<0.001^{*}$ & 0.25 & 0.080 & 0.37 & 0.010 \\
\hline Outer inferior sector & 0.42 & $<0.001^{*}$ & 0.17 & 0.240 & 0.27 & 0.060 \\
\hline Outer temporal sector & 0.40 & $<0.001^{*}$ & 0.37 & 0.010 & 0.40 & $<0.001^{*}$ \\
\hline Average thickness & 0.24 & 0.360 & 0.66 & $<0.001^{*}$ & 0.60 & 0.010 \\
\hline Macular volume & 0.34 & 0.160 & 0.41 & 0.080 & 0.34 & 0.160 \\
\hline \multicolumn{7}{|l|}{ RNFL thickness } \\
\hline Average & -0.42 & $<0.001^{*}$ & -0.58 & $<0.001^{*}$ & -0.56 & $<0.001^{*}$ \\
\hline Superior quadrant & -0.39 & $<0.001^{*}$ & -0.50 & $<0.001^{*}$ & -0.45 & $<0.001^{*}$ \\
\hline Nasal quadrant & -0.10 & 0.480 & -0.32 & 0.020 & -0.33 & 0.020 \\
\hline Inferior quadrant & -0.37 & $0.010^{*}$ & -0.46 & $<0.001^{*}$ & -0.44 & $<0.001^{*}$ \\
\hline Temporal quadrant & -0.25 & 0.080 & -0.21 & 0.140 & -0.28 & 0.050 \\
\hline
\end{tabular}

Abbreviations: ETDRS, Early Treatment Diabetic Retinopathy Study; RNFL, retinal nerve fiber layer; VA, visual acuity.

Data in bold type correspond to statistically significant correlations $(P$-value $<0.05)$. Asterisk indicates a significant difference by Pearson's test after Bonferroni's correction for multiple tests ( $P \leq 0.0045$ for macular thickness; $P \leq 0.010$ for RNFL thickness).

strongly associated with the Farnsworth S-index $(r=-0.75, P<0.001)$.

The VA ETDRS results correlated with macular and RNFL thickness: VA at $100 \%$ was significantly associated with all outer macular sectors (Table 4 ) and macular average thickness correlated with VA at $1.25 \%(r=0.60$,
$P=0.014)$ and $2.50 \%(r=0.66, P<0.001)$. VA was significantly correlated with average, superior, nasal, and inferior RNFL thickness at all contrast levels (Table 4).

No correlation was observed between disease severity (as measured by the MMSE) and ophthalmological parameters. 
Correlations between structural and functional measurements in controls can be observed in Supplementary Tables 1 and 2.

\section{Discussion}

In the present study, we evaluated visual function parameters and assessed the association between visual dysfunction and morphologic changes in the retina of 24 patients with AD. Parameters corresponding to CSV at different spatial frequencies were altered in AD patients compared with healthy subjects. Moreover, contrast sensitivity was the most affected parameter in our study and correlated with most of the structural data. Color vision was measured with two different tests, the Farnsworth and L'Anthony $15 \mathrm{D}$ tests. These tests provide more sensitive information than other tests for differentiating subjects with severe color vision loss from those with milder color defects or normal color vision, and can also be used to evaluate acquired loss of color vision. Both tests are color arrangement tests; however, L'Anthony color test is less saturated and therefore more suitable for detecting mild color anomalies. In our study, the AC CCI corresponding to both the Farnsworth and L'Anthony test was significantly altered in AD patients, corresponding to a worse arrangement of color caps in patients compared to controls. Farnsworth S-Index was also altered in AD subjects suggesting a mild tendency toward protanomaly.

Previous studies demonstrated alterations in sensory perception and visual processing in patients with AD. ${ }^{2,21-23}$ Contrast sensitivity (evaluated using the Sloan chart, computerized LogMAR ETDRS chart, frequencydoubling technology, and backward masking tests) disturbances are present even in the early stages of AD. ${ }^{24-26}$ Color vision, especially in the blue tone, is altered in these patients. ${ }^{27}$ Disease pathology in the precortical visual system is a possible mechanism underlying these visual impairments observed in AD patients. The parvo- and magnocellular ganglion cells are located in the RGC layer and result in two different pathways which identify color and contrast at different frequencies. ${ }^{28}$ The parvocellular RGCs are smaller and more numerous than other RGCs and result in the parvocellular pathway, which is specialized to identify patterns and color and is more sensitive to lower temporal frequency and higher spatial frequency. The magnocellular pathway originates in the magnocellular RGCs (larger and less numerous) and is more sensitive to higher temporal frequency and lower spatial frequency. A third set of cells, the koniocellular RGCs (larger than parvocellular RGCs), receives input from shortwave cones that are sensitive to blue-yellow tones. ${ }^{28-30}$ Previous studies (histologic, electroretinogram, and imaging studies) of these pathways suggest that the general loss of magnocellular and parvocellular cells is likely to be an important contributory mechanism for visual impairment in AD. ${ }^{31}$ Deficits specific to the magnocellular pathway have been identified in individuals with AD in the primary visual cortex and in brain areas devoid of plaques and neurofibrillary tangles. ${ }^{32}$ However, dendritic atrophy and loss of retinal ganglion cells have also been observed in the retina of a mouse model of AD, where the accumulation of beta-amyloid in the inner retinal layers was observed. This beta-amyloid deposits may be responsible for the depletion of parvo- and magnocellular cells in the retina. The loss of RGC was recently identified as the ultimate responsible for visual impairment in patients suffering from another neurodegenerative process, multiple sclerosis. ${ }^{33}$ Thus, a similar process could be the cause of contrast and color deficiencies in AD individuals.

Previous studies suggested that peripapillary RNFL, ${ }^{34}$ macular thickness and the macular inner retinal layers are affected in AD patients. ${ }^{5,7}$ Macular RNFL thickness and total macular volume measured by OCT have highly significant sensitivity and specificity for differentiating mild AD patients from healthy subjects. ${ }^{7}$ RNFL thickness has also been correlated with AD severity, and is predictive of axonal damage in these patients. ${ }^{35}$ Structural measurements, especially those corresponding to the macular area, were affected in our patients, consistent with previous reports. ${ }^{5,7}$ In addition and according to previous published research, our patients did not present significant reduction in foveal thickness. ${ }^{6,35,36}$ Patients with AD present with decreased visual acuity and contrast sensitivity vision, which depend on large extent on the foveal area. However, visual dysfunction in AD patients seems more likely to be caused by a general loss of the retinal ganglion cells, ${ }^{36,37}$ which ultimately leads to the alteration of the visual pathways responsible for contrast sensitivity and color vision. ${ }^{31,36}$ With the introduction of new OCT software for segmentation and analysis of the different retinal layers, recent studies have focused on the macular ganglion cell layer as responsible for the visual changes in $\mathrm{AD}$ and as a possible biomarker for disease progression and neural damage.

To the best of our knowledge, there are no previously published reports of a correlation between structural changes in the retina of AD patients and alterations observed in contrast sensitivity and color vision. We found that contrast sensitivity results (with both CSV-1000E and Pelli-Robson tests) and color vision (with both L'Anthony and Farnsworth tests) were significantly associated with structural changes, especially macular thickness (not with foveal thickness). Further studies including the analysis of the retinal ganglion cell complex would be needed to better understand the 
physiopathology of visual impairment in AD patients, and to determine the usefulness of visual function tests in combination with OCT measurements as a biomarker of severity and progression in AD.

\section{Summary}

What was known before

- Recent studies reported retinal thinning in Alzheimer's disease (AD) patients compared with healthy subjects. AD patients exhibit decreased color vision and contrast sensitivity, and other visual field deficits. Very few published studies have assessed visual dysfunction in AD and its correlation with morphologic parameters.

What this study adds

- Patients with AD have visual dysfunction that correlates with structural changes evaluated by spectral domainoptical coherence tomography. Macular measurements may be reliable indicators of visual impairment in AD patients.

\section{Conflict of interest}

The authors declare no conflict of interest.

\section{Acknowledgements}

This research was supported in part by the Fundación Mutua Madrileña grant FMMA 02/12. MS was supported by a fellowship from the Instituto de Salud Carlos III, Spain (Rio Hortega program: CM14/00026). All subjects gave detailed consent to participate in this study, which was conducted in accordance with the guidelines established by the Ethics Committee of the Miguel Servet Hospital and based on the principles of the Declaration of Helsinki. This research was supported in part by the Fundación Mutua Madrileña grant FMMA 02/12.

\section{References}

1 Blennow K, de Leon MJ, Zetterberg H. Alzheimer's disease. Lancet 2006; 368(9533): 387-403.

2 Cronin-Golomb A, Corkin S, Rizzo JF, Cohen J, Growdon JH, Banks KS. Visual dysfunction in Alzheimer's disease: relation to normal aging. Ann Neurol 1991; 29(1): 41-52 Erratum in: Ann Neurol 1991; 29(3): 271.

3 Cronin-Golomb A, Corkin S, Growdon JH. Visual dysfunction predicts cognitive deficits in Alzheimer's disease. Optom Vis Sci 1995; 72(3): 168-176.

4 Denise A, Valenti OD. Alzheimer's disease: Visual system review. Optometry 2010; 81: 12-21.

5 Blanks JC, Torigoe Y, Hinton DR, Blanks RH. Retinal pathology in Alzheimer's Disease I. Ganglion cell loss in foveal/parafoveal retina. Neurobiol Aging 1996; 17(3): 377-384.

6 Polo V, Garcia-Martin E, Bambo MP, Pinilla J, Larrosa JM, Satue $\mathrm{M}$ et al. Reliability and validity of Cirrus and Spectralis optical coherence tomography for detecting retinal atrophy in Alzheimer's disease. Eye (Lond) 2014; 28(6): 680-690.

7 Garcia-Martin ES, Rojas B, Ramirez AI, de Hoz R, Salazar JJ, Yubero R. Macular thickness as a potential biomarker of mild Alzheimer's disease. Ophthalmology 2014; 121(5): 1149-1151.

8 Valenti DA. Alzheimer's disease and glaucoma: imaging the biomarkers of neurodegenerative disease. Int J Alzheimers Dis 2011; 2010: 793931.

9 Gilmore GC, Levy JA. Spatial contrast sensitivity in Alzheimer's disease: a comparison of two methods. Optom Vis Sci 1991; 68: 790-794.

10 Neargarder SA, Stone ER, Cronin-Golomb A, Oross S 3rd. The impact of acuity on performance of four clinical measures of contrast sensitivity in Alzheimer's disease. J Gerontol B Psychol Sci Soc Sci 2003; 58: P54-P62.

11 Cronin-Golomb A. Vision in Alzheimer's disease. Gerontologist 1995; 35: 370-376.

12 Parisi V, Manni G, Spadaro M, Colacino G, Restuccia R, Marchi $\mathrm{S}$ et al. Correlation between morphological and functional retinal impairment in multiple sclerosis patients. Invest Ophthalmol Vis Sci 1999; 40: 2520-2527.

13 Kaur M, Saxena R, Singh D, Behari M, Sharma P, Menon V. Correlation between structural and functional retinal changes in Parkinson disease. J Neuroophthalmol 2015; 35(3): 254-258.

14 Parisi V, Restuccia R, Fattapposta F, Mina C, Bucci MG, Pierelli F. Morphological and functional retinal impairment in Alzheimer's disease patients. Clin Neurophysiol 2001; 112(10): 1860-1867.

15 American Psychiatric Association. Diagnostic and Statistical Manual of Mental Disorders (DSM-IV). 4th ed. American Psychiatric Association: Washington, DC, USA, 1994.

16 McKhann G, Drachman D, Folstein M, Katzman R, Price D, Stadlan EM. Clinical diagnosis of Alzheimer's disease: report of the NINCDS-ADRDA Work Group under the auspices of Department of Health and Human Services Task Force on Alzheimer's Disease. Neurology 1984; 34: 939-944.

17 Folstein MF, Folstein SE, McHugh PR. Mini-Mental State Examination: a practical method for grading the cognitive state of patients for the clinician. J Psychiatr Res 1975; 12: 189-198.

18 Vingrys AJ, King-Smith PE. A quantitative scoring technique for panel tests of color vision. Invest Ophthalmol Vis Sci 1988; 29(1): 50-63.

19 Bowman AJ. A method for quantitative scoring of the Farnsworth panel D15. Acta Ophthalmol (Copenh) 1982; 60: 907-916.

20 Schuman JS, Pedut-Kloizman T, Hertzmark E, Hee MR, Wilkins JR, Coker JG et al. Reproducibility of nerve fiber layer thickness measurements using optical coherence tomography. Ophthalmology 1996; 103(11): 1889-1898.

21 Thiyagesh SN, Farrow TFD, Parks RW, Accosta-Mesa H, Young C, Wilkinson ID et al. The neural basis of visuospatial perception in Alzheimer's disease and healthy elderly comparison subjects: an fMRI study. Psychiatry Res 2009; 172: 109-116.

22 Mapstone M, Dickerson K, Duffy CJ. Distinct mechanisms of impairment in cognitive ageing and Alzheimer's disease. Brain 2008; 131: 1618-1629.

23 Gilmore GC, Wenk HE, Naylor LA, Koss E. Motion perception and Alzheimer's disease. J Gerontol 1994; 49 : P52-P57.

24 Nolan JM, Loskutova E, Howard AN,, Moran R, Mulcahy R, Stack J et al. Macular pigment, visual function, and macular 
disease among subjects with Alzheimer's disease: an exploratory study. J Alzheimers Dis 2014; 42: 1191-1202.

25 Gilmore GC, Cronin-Golomb A, Neargarder SA, Morrison SR. Enhanced stimulus contrast normalizes visual processing of rapidly presented letters in Alzheimer's disease. Vision Res 2005; 45(8): 1013-1020.

26 Risacher SL, Wudunn D, Pepin SM, MaGee TR, McDonald BC, Flashman LA et al. Visual contrast sensitivity in Alzheimer's disease, mild cognitive impairment, and older adults with cognitive complaints. Neurobiol Aging 2013; 34(4): 1133-1144.

27 Cronin-Golomb A, Sugiura R, Corkin S, Growdon JH. Incomplete achromatopsia in Alzheimer's disease. Neurobiol Aging 1993; 14: 471-477.

28 Laycock R, Crewther SG, Crewther DP. A role for the 'magnocellular advantage' in visual impairments in neurodevelopmental and psychiatric disorders. Neurosci Biobehav Rev 2007; 31: 363-376.

29 Martin PR, White AJ, Goodchild AK, Wilder HD, Sefton AE. Evidence that blue-on cells are part of the third geniculocortical pathway in primates. Eur J Neurosci 1997; 9: 1536-1541.

30 Solomon SG, Lennie P. The machinery of colour vision. Nat Rev Neurosci 2007; 8: 276-286.

31 Kirby E, Bandelow S, Hogervorst E. Visual impairment in Alzheimer's disease: a critical review. J Alzheimers Dis 2010; 21(1): 15-34.
32 Hof P, Morrison J. Quantitative analysis of a vulnerable subset of pyramidal neurons in Alzheimer's disease: II. Primary and secondary visual cortex. J Comp Neurol 1990; 301: 55-64.

33 Lampert EJ, Andorra M, Torres-Torres R, Ortiz-Pérez S, Llufriu S, Sepúlveda $\mathrm{M}$ et al. Color vision impairment in multiple sclerosis points to retinal ganglion cell damage. J Neurol 2015; 262(11): 2491-2497.

34 Coppola G, Di Renzo A, Ziccardi L, Martelli F, Fadda A, Manni G et al. Optical Coherence Tomography in Alzheimer's Disease: A Meta-Analysis. PLoS One 2015; 10(8): e0134750

35 Larrosa JM, Garcia-Martin E, Bambo MP, Pinilla J, Polo V, Otin $S$ et al. Potential new diagnostic tool for Alzheimer's disease using a linear discriminant function for Fourier domain optical coherence tomography. Invest Ophthalmol Vis Sci 2014; 55: 3043-3051.

36 Marziani E, Pomati S, Ramolfo P, Cigada M, Giani A, Mariani $C$ et al. Evaluation of retinal nerve fiber layer and ganglion cell layer thickness in Alzheimer's disease using spectral-domain optical coherence tomography. Invest Ophthalmol Vis Sci 2013; 54(9): 5953-5958.

37 Garcia-Martin E, Bambo MP, Marques ML, Satue M, Otin S, Larrosa JM et al. Ganglion cell layer measurements correlate with disease severity in patients with Alzheimer's disease. Acta Ophthalmol 2016; 94(6): e454-e459.

Supplementary Information accompanies this paper on Eye website (http://www.nature.com/eye) 